\title{
Colagem de madeira de eucalipto com adesivos naturais
}

\author{
Eucalypts wood glue with \\ natural adhesives
}

\author{
Sabrina Barros Santiago ${ }^{1}$, Fabricio Gomes Gonçalves ${ }^{1}$, \\ Roberto Carlos Costa Lelis ${ }^{2}$, Pedro Gutemberg de Alcântara Segundinho ${ }^{1}$, \\ Juarez Benigno Paes ${ }^{1}$, Marina Donária Chaves Arantes ${ }^{3}$
}

\footnotetext{
${ }^{1}$ Departamento de Ciências Florestais e da Madeira, Centro de Ciências Agrárias e Engenharias, Universidade Federal do Espírito Santo, Av. Governador Lindemberg, 316, Centro, CEP 29.550-000, Jerônimo Monteiro, ES, Brasil.

e-mail: sabrina_santiago19@ hotmail.com, fabricio.goncalves@ufes.br, p_gutemberg2001@yahoo.com.br, jbp2@uol.com.br

${ }^{2}$ Departamento de Produtos Florestais, Instituto de Florestas, Universidade Federal Rural do Rio de Janeiro, Rod. BR 465, Km 07, CEP 23.890-000, Seropédica, RJ, Brasil.

e-mail: lelis@ufrrj.br

3 Departamento de Ciências Agrárias, Campus Sete Lagoas, Universidade Federal de São João del-Rei, Rodovia MG 424 - Km 47, CEP 35.701-970, Cx. Postal 56, Sete Lagoas, MG, Brasil.

e-mail: mdonariac@hotmail.com
}

\section{RESUMO}

Taninos da casca de eucaliptos foram obtidos por meio da extração com água sob adição de $8 \%$ de $\mathrm{Na}_{2} \mathrm{CO}_{3} \mathrm{e}$ a $4 \%$ de $\mathrm{Na}_{2} \mathrm{SO}_{4}$, com posterior formulação de adesivos. As propriedades adesivas foram avaliadas quanto a viscosidade, densidade, teor de sólidos, tempo de formação de gel e $\mathrm{pH}$ dos adesivos da acácia negra a 50\%, ureia $100 \%$, de tanino da casca de eucalipto extraído com $\mathrm{Na}_{2} \mathrm{CO}_{3}$ a $30 \%$ e de $\mathrm{Na}_{2} \mathrm{SO}_{4}$ a $50 \%$, e ureia com taninos extraídos das cascas de eucalipto na relação de 90:10 e 80:20. Resíduos madeireiros de eucalipto (classificados em três classes de densidade: baixa, média e alta) foram colados a quente com adesivos tânicos e sintético. A resistência das juntas coladas foi determinada por meio de resistência ao cisalhamento e falha na madeira. Pode-se concluir que os adesivos compostos pelo tanino extraído com sulfato de sódio e com carbonato de sódio puros (100\%) apresentaram características desejáveis para a produção de taninos, mas não o suficiente para sua utilização como adesivo em madeira em sua totalidade, podendo ser utilizado no enriquecimento de ureia-formaldeído na colagem da madeira. A maior resistência ao cisalhamento na madeira ocorreu na classe de densidade alta, para todos os adesivos empregados, enquanto que as maiores falhas na madeira na maioria dos adesivos testados ocorreram em madeira de baixa densidades.

Palavras-chave: Resíduos madeireiros; eucalipto; adesivos naturais; taninos; colagem a quente.

\section{ABSTRACT}

Tannins of eucalyptus bark were obtained by extracting with water under addition of $8 \% \mathrm{Na}_{2} \mathrm{CO}_{3}$ and $4 \%$ $\mathrm{Na}_{2} \mathrm{SO}_{4}$. To determine the adhesive properties was evaluated the viscosity, density, solids, gel time, and $\mathrm{pH}$ of adhesive of black wattle $50 \%$, urea $100 \%$, tannin extracted from the eucalyptus bark with $\mathrm{Na}_{2} \mathrm{CO}_{3} 30 \%$ and $\mathrm{Na}_{2} \mathrm{SO}_{4} 50 \%$, and urea with tannin extracted from the eucalyptus bark of ratio of 90:10 and 80:20. Waste eucalypts wood (density classified into three classes: low, medium and high) were glued to the hot tannic and synthetic adhesives. The resistance was determined by the glued joints shear strength and wood failure. It can be concluded that the adhesive compounds by tannin extracted with sodium sulphate and pure sodium carbonate $(100 \%)$ had desirable characteristics for the production of tannins, but not enough for its use as adhesive wood in its entirety and it may be used in the urea-formaldehyde glue gem enrichment in the timber. The higher shear strength on wood occurred in the high density class for all adhesives used, while larger gaps in wood adhesives in most tests it occurred in the wooden low densities.

Keywords: Waste timber; eucalypt; natural adhesives; tannins; hot glue. 


\section{INTRODUÇÃO}

A madeira é um material estrutural antigo e versátil, capaz de formar desde um lápis até grandes estruturas na construção de edificações, além de contribuir para o desenvolvimento sustentável. [1] evidenciam o quanto a madeira é valiosa, necessária e matéria-prima de uma gama de produtos disponíveis em nosso dia-a-dia. Dentre sua ampla utilidade, destacam-se os produtos madeireiros colados com adesivos, capaz de unir peças e obter diversas formas e aplicações.

Uma das principais vantagens da madeira colada é a possibilidade que peças de pequenas dimensões podem ser utilizadas como parte de laminações de vigas estruturais. Nas vigas de madeiras, variadas classes de resistência podem ser utilizadas e planejadas para que as peças com maiores resistências componham as partes mais externas e as de menor resistência a região interna da viga. Além do mais, pode-se também utilizar madeira de diferentes espécies na estrutura, conforme a solicitação de resistência das laminações em cada região da viga [2].

Nos processos tecnológicos, em várias unidades processadoras de madeira observa-se volume considerável de descarte de resíduos madeireiros que podem ser reaproveitados [3]. Por meio da colagem ocorre aumento considerável do uso de peças de pequenas dimensões, que minimizam a exploração das florestas, além de agregar maior valor econômico [4].

Para a colagem da madeira são utilizados adesivos sintéticos, contudo alguns são tóxicos para a saúde das pessoas. Pensando nisto, adesivos de fontes naturais como taninos têm sido explorados na comunidade científica, buscando ao menos a substituição parcial dos adesivos sintéticos. [5] A qualidade da colagem com taninos é influenciada pelos componentes químicos não fenólicos presentes nos extratos como açúcares, aminoácidos, pectinas [5], assim como por produtos químicos utilizados na extração de taninos a partir de diferentes plantas leguminosas [6].

Para a colagem, as condições físicas e químicas da superfície são importantes, com viscosidade adequada dos adesivos para umedecer e espalhar nas superfícies, e posterior pressão para melhor consolidar as juntas de madeira coladas. De forma geral, o adesivo deve ser capaz de fluir e preencher espaços vazios entre as juntas coladas, gerando interação entre o adesivo e madeira [7], desse modo, uma superfície rugosa pode aumentar a adesão entre as juntas coladas.

A densidade é uma característica intrínseca da madeira, e ao ser utilizada na composição de materiais aderidos por adesivos pode influenciar a resistência mecânica do produto formado. Em colagem de madeiras de elevada densidade, foi constatado que a degradação do mecanismo de adesão foi mais rápida que madeiras menos densas [8], havendo assim uma relação direta da densidade com a porosidade e permeabilidade da madeira, fatores influentes na formação da ligação adesivo-madeira.

Nesse contexto, o objetivo do trabalho consistiu em avaliar o comportamento dos adesivos de taninos extraídos das cascas de eucalipto, em juntas coladas de madeira de eucalipto em diferentes classes de densidades.

\section{MATERIAL E MÉTODOS}

\subsection{Origem, processamento e análises física e química da madeira}

Resíduos de cascas de eucalipto foram fornecidos por uma empresa de tratamento de madeira, localizada no município de Alegre, Sul do estado do Espírito Santo. O material foi seco ao ar (temperatura e umidade relativa do ar com médias de $21,3{ }^{\circ} \mathrm{C}$ e $74 \%$, respectivamente), até atingir a umidade de equilíbrio entre $12 \%$ e $15 \%$. Para uniformizar e acelerar o processo de secagem, o material foi periodicamente revolvido, para evitar perda de material.

Os taninos das cascas foram extraídos com soluções contendo carbonato de sódio $-\mathrm{Na}_{2} \mathrm{CO}_{3}$ a $8 \%$ e sulfato de sódio $-\mathrm{Na}_{2} \mathrm{SO}_{4}$ a $4 \%$, em autoclave por um período de 2 horas, numa relação licor:casca (15:1). Cada material foi submetido a duas sequências de extrações, a fim de se obter a máxima quantidade de extrativos. Após cada extração, o material foi filtrado em peneira de 270 mesh, colocado em bandejas de alumínio e disposto em estufa a $103{ }^{\circ} \mathrm{C} \pm 2{ }^{\circ} \mathrm{C}$ até secagem completa, na qual o material seco foi triturado em liquidificador industrial, obtendo-se o extrato na forma de pó. Procedimentos similares na extração de compostos tânicos foram realizados por alguns autores onde variou-se o sal extrator e ou tempo de extração, bem como a espécie [6, 24, 30, 47, 48].

A partir do tanino puro obtido formou-se uma solução de $50 \%$ com água destilada, para avaliação de suas propriedades tecnológicas: viscosidade, teor de sólidos, tempo de formação de gel, densidade e pH.

A viscosidade foi obtida por meio de viscosímetro digital com haste e rotação adequada ao material 
em 200 mL da solução. $\mathrm{O}$ pH da solução foi determinado por meio de pH-metro digital. O teor de sólidos, em porcentagem, foi determinado pela a razão da massa, antes e após secagem em estufa por aproximadamente 12 horas a $103^{\circ} \mathrm{C} \pm 2{ }^{\circ} \mathrm{C}[9]$.

O tempo de gel foi obtido com uma quantidade de adesivo disposta em um tubo de ensaio com catalisador (formaldeído $37 \%$ na proporção de $20 \%$ do teor de sólidos para as soluções de tanino puro; sulfato de amônia $24 \%$ na proporção de $2 \%$ sobre o teor de sólidos para o adesivo ureia formaldeído), homogeneizados em banho-maria $\left( \pm 90^{\circ} \mathrm{C}\right)$ até o ponto de endurecimento (mudança de fase).

Utilizaram-se picnômetros de $25 \mathrm{ml}$, em que após divisão da massa de adesivo existente em seu interior e respectiva divisão, obteve-se a densidade do adesivo $\left(\mathrm{g} \mathrm{cm}^{-1}\right)$, à temperatura ambiente $\left( \pm 27^{\circ} \mathrm{C}\right)[10]$.

Com o tanino extraído com carbonato de sódio $\left(\mathrm{Na}_{2} \mathrm{CO}_{3}\right)$ não foi possível realizar a diluição prevista de $50 \%$ como a do tanino extraído com sulfato de sódio, pois tal quantidade de solvente não hidratou o suficiente para proporcionar viscosidade possível de ser determinada. Portanto, utilizou-se a solução com $70 \%$ de água e $30 \%$ de tanino. Com o propósito de melhorar a viscosidade, a solução foi mantida em uma manta aquecedora (aproximadamente $40^{\circ} \mathrm{C}$ ) por 30 minutos.

As propriedades adesivas foram obtidas dos resíduos da casca de eucalipto extraídos com carbonato de sódio $(8 \%)$ e sulfato de sódio (4\%). Utilizou-se o extrato tânico extraído (100\%), assim como sua substituição em dois níveis (10 e 20\%) nos adesivos de ureia-formaldeído. Para efeito de comparação utilizou-se também a ureia formaldeído (100\%) e tanino industrial de acácia negra (100\%). Desta forma, os adesivos e proporções utilizados são: ureia (100\%), tanino de acácia negra (100\%), tanino extraído com sulfato de sódio (100\%), tanino extraído com carbonato de sódio (100\%), tanino extraído com sulfato de sódio:ureia (10:90), tanino extraído com sulfato de sódio:ureia (20:80), tanino extraído com carbonato de sódio:ureia (10:90) e tanino extraído com carbonato de sódio:ureia (20:80).

\subsection{Produção das juntas de madeira}

As amostras de madeira utilizadas para a colagem dos diferentes adesivos foram obtidas a partir do resíduo do processamento de diferentes espécies de eucalipto a partir dos desdobros primários e secundários (costaneira, refilo e apara) de várias dimensões, utilizadas para obtenção de janelas, portas, móveis e de peças estruturais no município de Jerônimo Monteiro, Sul do estado do Espírito Santo.

A densidade de cada resíduo de madeira foi determinada conforme a Norma Brasileira Regulamentadora - ABNT NBR 11941 [11]. Assim, foram confeccionados cinco corpos de prova $(2 \times 2 \times 2 \mathrm{~cm})$ de cada resíduo de madeira para posterior saturação sob vácuo por 30 dias. Depois de saturados, determinou-se o volume dos mesmos pelo método de deslocamento de massa em água [12].

A massa anidra foi determinada por meio da submissão de amostras colocadas em estufa à temperatura de $103{ }^{\circ} \mathrm{C} \pm 2{ }^{\circ} \mathrm{C}$, com monitoramento até que a massa permanecesse constante (variação inferior a $1 \%$ ) e a pesagem foi realizada em balança de precisão $(0,01 \mathrm{~g})$. As amostras de madeira foram classificadas em três classes de densidade, baixa $\left(\leq 0,50 \mathrm{~g} \mathrm{~cm}^{-3}\right)$; média $\left(0,5\right.$ a $\left.0,72 \mathrm{~g} \mathrm{~cm}^{-3}\right)$, alta $\left(>0,72 \mathrm{~g} \mathrm{~cm}^{-3}\right)$, para melhor controle dos efeitos gerados da interação madeira adesivo [13].

As amostras de madeira foram redimensionadas em peças com 0,6 $\times 2,60 \times 43 \mathrm{~cm}$ (espessura $\times$ largura $\times$ comprimento) obedecendo à orientação dos elementos anatômicos nos sentidos radiais e tangenciais, para melhor processo de colagem das lamelas de madeira. Posteriormente climatizadas $\left(25^{\circ} \mathrm{C} \pm 2{ }^{\circ} \mathrm{C}\right.$ e $65 \% \pm$ $5 \%$ de umidade relativa - UR), até atingirem umidade de equilíbrio, e com sucessivo monitoramento para evitar o desenvolvimento de fungos e agentes xilófagos.

Foram determinados o teor de extrativos totais, lignina e cinzas (nas três classes de densidade) da madeira empregada para a colagem. O teor de extrativos totais foi obtido ao serem utilizados 2,0 g (base seca) de casca alocadas em filtro de vidro sintetizado no extrator Soxhlet junto com uma solução álcool:tolueno (1:2 v.v.) por 5 horas, álcool por 4 horas e em água quente por 1 hora. Os solventes foram evaporados em estufa a $40{ }^{\circ} \mathrm{C}-60{ }^{\circ} \mathrm{C}$ e os resíduos obtidos foram pesados, obtendo-se o teor de extrativos por diferença de massa [14].

Com as amostras livres de extrativos realizou-se a análise do teor de lignina, com $0,3 \mathrm{~g}$ da amostra foi submetida à hidrólise com ácido sulfúrico $72 \%$ por 60 minutos $\left(30^{\circ} \mathrm{C} \pm 1{ }^{\circ} \mathrm{C}\right)$, autoclavada por 60 minutos e filtrada. O sólido retido foi considerado como a lignina insolúvel, a qual foi determinada por diferença de massa entre a madeira livre de extrativos [15].

O líquido remanescente da filtração da lignina insolúvel foi analisado em espectrofotômetro UV, para determinação da lignina solúvel [45]. O teor de lignina total consistiu na soma das frações solúvel e insolúvel. A holocelulose foi determinada pela diferença entre a massa inicial e a quantidade de extrativos e lignina 
total. O teor de cinza da casca foi obtido pelo método M-11/77 [17] com cinco repetições.

$\mathrm{O}$ pH e capacidade tampão foram determinados por meio da solubilização de 5,0 g de serragem (base seca), em $150 \mathrm{~mL}$ de água destilada, sob refluxo $\left(100^{\circ} \mathrm{C}\right)$ por 20 minutos, e à temperatura ambiente $\left(25^{\circ} \mathrm{C} \pm\right.$ $2{ }^{\circ} \mathrm{C}$ ) alocados na mesa agitadora por 24 horas. Posteriormente, foram pipetados $50 \mathrm{~mL}$ de extratos aquosos para obtenção do $\mathrm{pH}$. Na mesma solução, realizou-se uma titulação com solução de $\mathrm{NaOH} 0,001 \mathrm{~N}$ até elevar o pH a 7,0, e determinou-se a capacidade tampão ácida em mmol L-1 e convertidos em mmol NaOH $5 \mathrm{~g}^{-1}$ [18].

\subsection{Colagem e ensaio de cisalhamento}

As juntas de madeira foram formadas (total de 12 para cada tratamento) com lamelas para as três classes de densidade. Para cada junta de lamelas foram distribuídos adesivos na gramatura de $250 \mathrm{~g} \mathrm{~m}^{-2}$, aplicadas com uma espátula. Em seguida, prensadas (1,2 MPa) por um período de 8 minutos a $150{ }^{\circ} \mathrm{C}$, em prensa hidráulica, com aquecimento em ambos pratos, com posterior climatização durante 15 dias $\left(25^{\circ} \mathrm{C} \pm 2{ }^{\circ} \mathrm{C}\right.$ e $65 \% \pm 5 \%$ UR).

A resistência à linha de cola foi avaliada por meio de testes de cisalhamento das juntas coladas (American Society for Testing and Materials - ASTM D-2339-98 [19]. As dimensões dos corpos de prova foram de $0,5 \times 2,54 \times 8,26 \mathrm{~cm}$ (espessura $\times$ largura $\times$ comprimento). $\mathrm{O}$ ensaio de cisalhamento por tração das juntas coladas ocorre por meio da pressão, tensão, ou falha resultante das forças aplicadas, que tende a causar nos planos adjacentes da amostra, com deslize paralelo em direções opostas. O ensaio foi realizado em máquina com tomada automatizada de dados, de capacidade de $100 \mathrm{kN}$.

Para a avaliação da colagem, utilizou-se parâmetros de classificação da resistência ao cisalhamento (MPa) na linha de cola: muito baixo $(<4,0)$, baixo $(4,0-8,5)$, mediano $(8,6-12,0)$, alto $(12,1-17,5)$ e muito alto $(>17,5)$ [20].

A falha na madeira foi determinada com uso de uma película transparente quadriculada (10 linhas $x$ 10 colunas) disposta sobre a área cisalhada, conforme ASTM - D 5751-99 [21]. O percentual de falha na madeira foi avaliado pela média de falha e a resistência avaliada pelos critérios da ABNT NBR 7190 [17], a qual estabelece que a resistência média das juntas coladas deve ser no mínimo igual à da madeira ao cisalhamento, no teor de umidade padrão de $12 \%$. Para isto, os resultados obtidos nos ensaios foram ajustados (Equação 1).

$F_{12}=F_{u \%}\left[1+\frac{3(U \%-12)}{100}\right]$

em que: $\mathrm{F}_{12}=$ valor da resistência no teor de $12 \%$ de umidade; $\mathrm{F}_{\mathrm{U}(\%)}=$ valor da resistência encontrado no teor de umidade ensaiado; e U\% = umidade do corpo de prova no momento do ensaio.

Para a eficiência da colagem, foi utilizado o delineamento inteiramente casualisado em esquema fatorial $(3 \times 8)$, com a densidade da madeira em três níveis (baixa, média e alta) e o adesivo em oito níveis, sendo sete repetições cada tratamento. Análise de variância foi realizada, a fim de verificar a existência de diferença significativa entre os tratamentos, e em caso de existência de diferenças significativas, aplicou-se teste de Tukey $(\mathrm{p}<0,05)$ para comparação das médias. Anteriormente à análise, foram aplicados os testes de normalidade (Kolmorogov-Sminov) e de homogeneidade de variâncias (Kruskal-Wallis).

\section{RESULTADOS}

\subsection{Avaliação das propriedades dos adesivos}

Para os diferentes adesivos (Tabela 1) a análise variância foi significativa para a viscosidade, $\mathrm{pH}$, densidade $\mathrm{e}$ teor de sólidos. Os adesivos mais viscosos foram o tanino extraído de sulfato de sódio, assim como sua composição na proporção 80:20 com ureia. Para o adesivo extraído por carbonato de sódio a viscosidade foi a menor encontrada $(1044,66 \mathrm{cP})$, tal característica pode ser evidenciada pelo baixo teor de sólidos nos adesivos.

Nota-se que os valores de $\mathrm{pH}$ dos adesivos variaram da faixa ácida à básica, com comportamento básico encontrado para tanino extraído com carbonato de sódio e em suas misturas com ureia nas proporções 90:10 e 80:20 (ureia: tanino) com mesmo sal, não possuindo diferença estatística entre ambos. A presença de tanino extraído com carbonato de sódio pode apresentar comportamento semelhante ao adesivo sintético fenol formaldeído, por causa do seu $\mathrm{pH}$, que proporciona melhoria na colagem em madeiras de caráter próximos ao alcalino.

Contudo, observa-se que a madeira de eucalipto nas três densidades possui pH na faixa ácida, com 
maior dificuldade para cura com tanino extraído com carbonato de sódio. Comportamento diferente foi observado para tanino extraído com sulfato de sódio $(3,98)$, pois o mesmo encontra-se na faixa ácida, assim como o tanino comercial $(3,35)$, e ambos estão dentro da faixa de $\mathrm{pH}$ da madeira de eucalipto utilizadas nas três classes de densidade. Desta forma, esperam-se melhores resultados na colagem em madeira com esses adesivos.

Tabela 1: Valores médios da viscosidade, $\mathrm{pH}$, densidade, tempo de gel e teor de sólidos para os adesivos utilizados.

\begin{tabular}{|c|c|c|c|c|c|c|c|c|}
\hline \multirow[b]{2}{*}{ PROPRIEDADES } & \multicolumn{8}{|c|}{ ADESIVOS } \\
\hline & Ureia & $\begin{array}{c}\text { Tanino } \\
\text { industrial }^{\star *}\end{array}$ & $\begin{array}{c}\text { Tanino } \\
\left(\mathrm{Na}_{2} \mathrm{SO}_{4}\right)\end{array}$ & $\begin{array}{c}\text { Tanino } \\
\left(\mathrm{Na}_{2} \mathrm{CO}_{3}\right)\end{array}$ & $\begin{array}{l}\mathrm{U}: \mathrm{T}_{\mathrm{Na} 2 \mathrm{CO} 3} \\
(90: 10)\end{array}$ & $\begin{array}{l}\mathrm{U}: \mathrm{T}_{\mathrm{Na} 2 \mathrm{CO} 3} \\
(80: 20)\end{array}$ & $\begin{array}{c}\mathrm{U}: \mathrm{T}_{\mathrm{Na}} \mathrm{SO} \\
(90: 10)\end{array}$ & $\begin{array}{c}\mathrm{U}: \mathrm{T}_{\mathrm{Na} 2 \mathrm{SO} 4} \\
(80: 20)\end{array}$ \\
\hline Viscosidade (cp) & $\begin{array}{c}1845,04 \mathrm{c}^{\#} \\
(44,21)\end{array}$ & $\begin{array}{c}1409,36 \mathrm{~d} \\
(64,32)\end{array}$ & $\begin{array}{c}3331,56 \mathrm{a} \\
(188,48) \\
\end{array}$ & $\begin{array}{c}1044,66 \mathrm{e} \\
(35,65)\end{array}$ & $\begin{array}{c}1286,82 \mathrm{~d} \\
(21,57)\end{array}$ & $\begin{array}{c}1437,68 \mathrm{~d} \\
(19,57)\end{array}$ & $\begin{array}{c}2337,6 \mathrm{~b} \\
(68,83) \\
\end{array}$ & $\begin{array}{c}3387,7 \mathrm{a} \\
(80,35) \\
\end{array}$ \\
\hline $\mathrm{pH}$ & $\begin{array}{l}8,05 \mathrm{c} \\
(0,04)\end{array}$ & $\begin{array}{l}3,35 \mathrm{~g} \\
(0,02)\end{array}$ & $\begin{array}{l}3,98 \mathrm{f} \\
(0,02)\end{array}$ & $\begin{array}{l}9,59 \mathrm{a} \\
(0,02) \\
\end{array}$ & $\begin{array}{l}9,29 \mathrm{~b} \\
(0,04) \\
\end{array}$ & $\begin{array}{l}9,67 \mathrm{a} \\
(0,04)\end{array}$ & $\begin{array}{l}5,72 \mathrm{~d} \\
(0,08) \\
\end{array}$ & $\begin{array}{l}5,41 \mathrm{e} \\
(0,05) \\
\end{array}$ \\
\hline $\begin{array}{l}\text { Densidade } \\
\left(\mathrm{g} \mathrm{cm}^{-3}\right)\end{array}$ & $\begin{array}{l}1,37 \mathrm{~d} \\
(0,00)\end{array}$ & $\begin{array}{l}1,33 \mathrm{f} \\
(0,00)\end{array}$ & $\begin{array}{l}1,51 \mathrm{a} \\
(0,00)\end{array}$ & $\begin{array}{l}1,27 \mathrm{~g} \\
(0,01)\end{array}$ & $\begin{array}{l}1,35 \mathrm{e} \\
(0,01)\end{array}$ & $\begin{array}{l}1,34 \mathrm{e} \\
(0,00)\end{array}$ & $\begin{array}{l}1,48 \mathrm{c} \\
(0,00)\end{array}$ & $\begin{array}{l}1,49 \mathrm{~b} \\
(0,00)\end{array}$ \\
\hline Tempo de gel (s) & $\begin{array}{c}79,8 \\
(0,07) \\
\end{array}$ & $\begin{array}{l}297,48 \\
(0,49) \\
\end{array}$ & $>2700^{*}$ & $>2700^{*}$ & $\begin{array}{l}225,9 \\
(1,47) \\
\end{array}$ & $\begin{array}{c}780 \\
(0,30) \\
\end{array}$ & $\begin{array}{c}66 \\
(0,04) \\
\end{array}$ & $\begin{array}{c}66,3 \\
(0,01) \\
\end{array}$ \\
\hline $\begin{array}{l}\text { Teor de sólidos } \\
(\%)\end{array}$ & $\begin{array}{c}63,23 \mathrm{~b} \\
(0,34) \\
\end{array}$ & $\begin{array}{c}49,71 \mathrm{e} \\
(0,03)\end{array}$ & $\begin{array}{c}49,76 \mathrm{e} \\
(0,91) \\
\end{array}$ & $\begin{array}{l}31,22 \mathrm{f} \\
(0,18)\end{array}$ & $\begin{array}{c}58,42 \mathrm{~d} \\
(0,57)\end{array}$ & $\begin{array}{c}60,00 \mathrm{c} \\
(0,00)\end{array}$ & $\begin{array}{c}59,26 \mathrm{~cd} \\
(0,40) \\
\end{array}$ & $\begin{array}{c}65,33 \mathrm{a} \\
(0,24) \\
\end{array}$ \\
\hline
\end{tabular}

${ }^{\#}$ Médias seguidas pela mesma letra na linha não diferem estatisticamente (Tukey, $\left.p>0,05\right)$; ${ }^{*}$ Tempo de gel superior a $2700 \mathrm{~s}$ (45 min.) não foi possível determinar; U:ureia; T:tanino. ** Tanino em pó de acácia negra. cP - centipoise. Valor entre parênteses corresponde ao desvio padrão.

Os adesivos que se encontram na faixa ácida de $\mathrm{pH}$ são o tanino extraído com sulfato de sódio e nas proporções 90:10 e 80:20 de tanino e ureia com mesmo sal, assim como o tanino comercial. Enquanto que em faixa básica de pH encontram-se a ureia (100\%) e o tanino extraído com carbonato de sódio e nas proporções 90:10 e 80:20 de ureia e tanino.

O tanino extraído com sulfato de sódio obteve maior densidade em relação aos demais adesivos. Enquanto o menor valor encontrado foi para extrato tânico de carbonato de sódio, por apresentar somente $30 \%$ de taninos em sua formulação, valor baixo em virtude de sua elevada viscosidade. O extrato tânico obtido com sulfato de sódio proporcionou maior densidade para adesivos de mistura de ureia e tanino nas proporções avaliada (10 e 20\%). Desse modo, pode-se inferir que a casca de eucalipto apresenta compostos químicos de grande peso molecular, e consequentemente, proporciona adesivos de maiores densidades.

Por meio da estatística descritiva, observou-se, menor tempo de gel para o tanino extraído com sulfato de sódio e nas misturas de ureia e tanino nas proporções 90:10 e 80:20. Normalmente, a cura da resina ureia formaldeído - UF é acelerada à medida que o pH é diminuído. Por isso, acrescenta-se à resina um catalisador, que libera ácido como, por exemplo, o sulfato de amônio.

Os extratos tânicos de carbonato e sulfato de sódio apresentaram caráter básico e ácido respectivamente. Consequentemente, o pH da ureia nas diferentes proporções de tanino foi alterado. Contudo, para os extratos tânicos de carbonato e sulfato de sódio não foi possível identificar o tempo exato da mudança de fase para o gel que extrapolou $2700 \mathrm{~s}$ (45 min), ao empregar o formaldeído como catalisador, enquanto nas demais formulações, que continham ureia.

O teor de sólidos apresentou maior valor obtido na proporção 80:20, de extrato tânico contendo sulfato de sódio e ureia, seguido da ureia (100\%), evidenciando-se, portanto, a contribuição do tanino no aumento do teor de sólidos da composição deste adesivo.

Com aumento da viscosidade das soluções de tanino há geralmente incremento do $\mathrm{pH}$. Mas para alguns tipos de taninos não há clara dependência da viscosidade com o pH. Assim, para o extrato tânico contendo sulfato de sódio e ureia na proporção de 80:20 ocorreu maior viscosidade.

De modo geral, a alteração na formulação do adesivo ureia com adição de tanino extraído com solução de sulfato de sódio proporcionou aumento do teor de sólidos, da densidade e viscosidade, e diminuição do tempo de gel, tais características adesivas são relativamente satisfatórias para posterior colagem de madeira. 


\subsection{Caracterização química da madeira}

No processo de colagem os constituintes químicos da madeira (Tabela 2) também podem interferir. Os teores de extrativos, ligninas, holocelulose e cinzas da madeira nas três classes de densidade foram significativos.

Tabela 2: Valores médios da composição química da madeira nas três classes de densidade.

\begin{tabular}{l|c|c|c|c}
\hline $\begin{array}{c}\text { Densidade da } \\
\text { madeira }\end{array}$ & Extrativos totais & Lignina total & Holocelulose & Teor de cinzas \\
\hline \multirow{2}{*}{ Baixa } & $2,50 \mathrm{c}^{\#}$ & $28,51 \mathrm{~b}$ & $68,98 \mathrm{a}$ & $2,31 \mathrm{a}$ \\
& $(0,34)$ & $(0,34)$ & $(0,49)$ & $(0,11)$ \\
\hline \multirow{2}{*}{ Média } & $4,96 \mathrm{~b}$ & $30,30 \mathrm{a}$ & $64,72 \mathrm{c}$ & $1,15 \mathrm{~b}$ \\
& $(0,27)$ & $(0,21)$ & $(0,12)$ & $(0,10)$ \\
\hline \multirow{2}{*}{ Alta } & $5,84 \mathrm{a}$ & $26,20 \mathrm{c}$ & $67,95 \mathrm{~b}$ & $0,93 \mathrm{c}$ \\
& $(0,40)$ & $(0,53)$ & $(0,70)$ & $(0,06)$ \\
\hline
\end{tabular}

\# Médias seguidas pela mesma letra na coluna não diferem estatisticamente (Tukey, p > 0,05). Valor entre parênteses corresponde ao desvio padrão.

Das constituições químicas da madeira, o teor de extrativos é um dos parâmetros importantes para efetivar uma boa colagem, deste modo, observou-se que madeiras de alta densidade apresentaram maior deposição de extrativos no tecido lenhoso, seguida das de média, e em menor quantidade nas de baixa densidade. Para o teor de lignina total, o maior valor médio ocorreu em madeira de média, e menor em madeira de alta densidade, enquanto que maiores teores de holocelulose ocorreu em madeira de alta densidade. Maiores teores de cinzas ocorreram em madeira de baixa densidade, seguidas pela média e alta densidades, podendose inferir que com aumento da densidade ocorre menores deposições de material inorgânico.

Nas três diferentes classes de densidade da madeira, foi observado diferença estatística nos valores de pH. A madeira de alta densidade, tanto em temperatura ambiente, quanto em ambiente aquecido a $100^{\circ} \mathrm{C}$, apresentou menor acidez, quando comparada com madeira das classes de média e baixa densidade (Tabela 3 ). Contudo, as médias das três classes de densidades estão agrupadas na faixa de $\mathrm{pH}$ ácido, por apresentar $\mathrm{pH}$ inferior a 7 .

Tabela 3: Valores médios de pH e capacidade tampão ácida para madeira de eucalipto em função da temperatura e densidade da madeira.

MADEIRA DE EUCALIPTO

\begin{tabular}{l|c|c|c|c}
\hline \multirow{2}{*}{ Densidade da madeira } & \multicolumn{2}{|c|}{ pH } & \multicolumn{2}{c}{ Capacidade tampão } \\
\cline { 2 - 5 } & Ambiente & Aquecida & Ambiente & Aquecida \\
\hline \multirow{2}{*}{ Baixa } & $4,18 \mathrm{c}^{\#}$ & $3,92 \mathrm{~b}$ & 0,061 & $0,103 \mathrm{c}$ \\
& $(0,15)$ & $(0,08)$ & $(0,00)$ & $(0,00)$ \\
\hline \multirow{2}{*}{ Média } & $4,37 \mathrm{~b}$ & $3,94 \mathrm{~b}$ & 0,092 & $0,157 \mathrm{~b}$ \\
& $(0,05)$ & $(0,12)$ & $(0,06)$ & $(0,01)$ \\
\hline \multirow{2}{*}{ Alta } & $4,61 \mathrm{a}$ & $4,23 \mathrm{a}$ & 0,127 & $0,205 \mathrm{a}$ \\
& $(0,02)$ & $(0,06)$ & $(0,01)$ & $(0,00)$ \\
\hline
\end{tabular}

\# Médias seguidas pela mesma letra na coluna não diferem estatisticamente (Tukey, $\mathrm{p}$ $>0,05)$ * Não significativo pelo teste $\mathrm{F}(\mathrm{p}>0,05)$. Valor entre parênteses corresponde ao desvio padrão.

A capacidade tampão ácida é um indicador utilizado para mensurar a capacidade que a solução possui em resistir a variação de pH. Ao titular a solução tampão em função das três diferentes classes de densidades, observa-se que na água em temperatura ambiente não houve diferença estatística. No entanto, comportamento diferente foi observado em água aquecida, tornando-se necessário menor quantidade de solução mmol $\mathrm{NaOH} 5 \mathrm{~g}^{-1}$ para neutralizar o $\mathrm{pH}$ da madeira de baixa densidade em relação às de média e alta densidades submetidas ao mesmo modo de extração.

\subsection{Ensaio de cisalhamento das juntas de madeira coladas}

Ao realizar a colagem e posterior prensagem das lamelas de madeira com os adesivos obtidos de taninos puros, provenientes das cascas de eucalipto, observou-se que ocorreu a delaminação das lamelas coladas, não 
sendo possível a realização do ensaio de cisalhamento; contudo, quando utilizados em diferentes concentrações (10 e 20\%) em misturas com ureia (90 e 80 \%) não ocorreu a delaminação após a climatização do material.

Para a análise de variância verificou-se que a Interação adesivo $\times$ densidade foi significativa $(\mathrm{p}<0,05)$. A comparação entre as médias para a resistência ao cisalhamento por tração para os seis de adesivos e classes de densidade da madeira constam da Tabela 4.

Tabela 4: Resistência ao esforço de cisalhamento por tração em lamelas de madeira de eucalipto coladas com diferentes adesivos em três densidades.

\section{RESISTÊNCIA AO CISALHAMENTO (MPa)}

\begin{tabular}{|c|c|c|c|c|c|c|}
\hline $\begin{array}{c}\text { Densidade da } \\
\text { madeira }\end{array}$ & Ureia & $\begin{array}{c}\text { Tanino } \\
\text { Industrial }\end{array}$ & $\begin{array}{c}\mathrm{U}: \mathrm{T}_{\mathrm{Na} 2 \mathrm{CO}} \\
(90: 10)\end{array}$ & $\begin{array}{c}\mathrm{U}: \mathrm{T}_{\mathrm{Na} 2 \mathrm{CO} 3} \\
(80: 20)\end{array}$ & $\begin{array}{c}\mathrm{U}: \mathrm{T}_{\mathrm{Na2SO4}} \\
(90: 10)\end{array}$ & $\begin{array}{c}\mathrm{U}: \mathrm{T}_{\mathrm{Na2SO}} \\
(80: 20)\end{array}$ \\
\hline Baixa & $\begin{array}{c}0,87 \mathrm{c} \mathrm{B}^{\#} \\
(2,27)\end{array}$ & $\begin{array}{c}1,02 \mathrm{~b} \mathrm{~B} \\
(15,48)\end{array}$ & $\begin{array}{c}2,13 \mathrm{~b} \mathrm{~A} \\
(6,13)\end{array}$ & $\begin{array}{c}1,05 \mathrm{~b} \mathrm{~B} \\
(2,36)\end{array}$ & $\begin{array}{c}0,98 \text { c B } \\
(1,64)\end{array}$ & $\begin{array}{c}1,32 \mathrm{~b} \mathrm{AB} \\
(4,64)\end{array}$ \\
\hline Média & $\begin{array}{c}1,96 \mathrm{~b} \mathrm{BC} \\
(6,84)\end{array}$ & $\begin{array}{c}4,20 \text { a A } \\
(14,58)\end{array}$ & $\begin{array}{c}2,50 \mathrm{~b} \mathrm{~B} \\
(8,79)\end{array}$ & $\begin{array}{c}1,47 \mathrm{~b} \mathrm{C} \\
(4,10)\end{array}$ & $\begin{array}{c}2,66 \mathrm{~b} \mathrm{~B} \\
(10,20)\end{array}$ & $\begin{array}{c}2,09 \text { a BC } \\
(8,48)\end{array}$ \\
\hline Alta & $\begin{array}{c}3,77 \text { a A } \\
(12,79)\end{array}$ & $\begin{array}{c}3,96 \text { a A } \\
(3,50)\end{array}$ & $\begin{array}{c}3,59 \text { a A } \\
(10,75)\end{array}$ & $\begin{array}{c}2,37 \text { a A } \\
(9,39)\end{array}$ & $\begin{array}{c}3,50 \text { a A } \\
(10,90)\end{array}$ & $\begin{array}{c}2,03 \text { a } \mathrm{A} \\
(5,64)\end{array}$ \\
\hline
\end{tabular}

\# Médias seguidas da mesma letra minúscula na coluna e maiúscula na linha não diferem estatisticamente (Tukey, $p>0,05$ ). Valor entre parênteses corresponde ao desvio padrão.

De modo geral, a densidade da madeira influenciou na adesão das juntas nos seis adesivos testados, atingindo o maior valor para resistência ao cisalhamento na maior classe de densidade, com valor máximo de 4,20 Mpa (Tabela 4). Nas três classes de densidade, a resistência ao cisalhamento foi muito baixa, com exceção da madeira de média densidade colada com tanino industrial. Os adesivos ureia (100\%), tanino comercial e as misturas de ureia com taninos nas proporções 90:10 e 80:20, não apresentaram diferença significativa na classe de alta densidade.

\subsection{Falha na madeira das juntas coladas}

$\mathrm{Na}$ análise de variância verificou-se que a Interação adesivo $x$ densidade foi significativa $(\mathrm{p}<0,05)$. A comparação entre as médias para a falha na madeira para os seis de adesivos e classes de densidade da madeira constam da Tabela 5 .

Tabela 5: Falha na madeira submetida ao esforço de cisalhamento para os adesivos e classes de densidade da madeira.

\begin{tabular}{|c|c|c|c|c|c|c|}
\hline \multicolumn{7}{|c|}{ FALHA NA MADEIRA (\%) } \\
\hline Densidade da madeira & Ureia & $\begin{array}{c}\text { Tanino } \\
\text { Comercial }\end{array}$ & $\begin{array}{c}\mathrm{U}: \mathrm{T}_{\mathrm{Na2CO}} \\
(90: 10)\end{array}$ & $\begin{array}{c}\mathrm{U}: \mathrm{T}_{\mathrm{Na2CO}} \\
(80: 20)\end{array}$ & $\begin{array}{c}\mathrm{U:T} \mathrm{T}_{\mathrm{Na2SO}} \\
(90: 10)\end{array}$ & $\begin{array}{c}\text { U:T } \\
(80: 20)\end{array}$ \\
\hline Baixa & $\begin{array}{c}88,71 \text { a } \mathrm{AB}^{\#} \\
(30,94)\end{array}$ & $\begin{array}{c}100,00 \text { a } \mathrm{A} \\
(0,00)\end{array}$ & $\begin{array}{c}43,28 \text { a B } \\
(33,69)\end{array}$ & $\begin{array}{c}95,71 \text { a A } \\
(26,32)\end{array}$ & $\begin{array}{c}100,00 \text { a } \mathrm{A} \\
(7,28)\end{array}$ & $\begin{array}{c}88,57 \text { a } A B \\
(42,25)\end{array}$ \\
\hline Média & $\begin{array}{c}32,71 \mathrm{~b} \mathrm{AB} \\
(32,14)\end{array}$ & $\begin{array}{c}77,85 \text { a A } \\
(33,53)\end{array}$ & $\begin{array}{c}14,00 \text { a B } \\
(6,55)\end{array}$ & $\begin{array}{c}13,57 \text { c B } \\
(7,49)\end{array}$ & $\begin{array}{c}20,85 \text { b B } \\
(33,15)\end{array}$ & $\begin{array}{c}57,85 \text { a } \mathrm{AB} \\
(40,71)\end{array}$ \\
\hline Alta & $\begin{array}{c}66,71 \text { ab } A B \\
(34,15)\end{array}$ & $\begin{array}{c}86,42 \text { a } A \\
(22,75)\end{array}$ & $\begin{array}{c}25,00 \text { a BC } \\
(32,05)\end{array}$ & $\begin{array}{c}53,71 \mathrm{~b} \mathrm{AB} \\
(37,06)\end{array}$ & $\begin{array}{c}58,42 \text { b AB } \\
(32,40)\end{array}$ & $\begin{array}{c}6,85 \mathrm{~b} \mathrm{C} \\
(5,63)\end{array}$ \\
\hline
\end{tabular}

\# Médias seguidas da mesma letra minúscula na coluna e maiúscula na linha não diferem estatisticamente (Tukey, p > $0,05)$. Valor entre parênteses corresponde ao desvio padrão.

Os maiores valores de falhas na madeira de eucalipto ocorreram nas juntas coladas de baixa densidade, com exceção da aderida com adesivo que continha extrato tânico com carbonato de sódio e ureia (10:90), as falhas na madeira atingiram o limite de $60 \%$. As madeiras de média densidade, utilizando adesivo de tanino industrial, e de elevada densidade, com uso dos adesivos ureia e tanino industrial também atingiram o limite preconizado pela normativa da ASTM - D 5751-99 [21]. Esse resultado indica maior segurança destes adesivos em relação aos demais quanto à resistência ao cisalhamento para a colagem da madeira em função das 
classes de densidades utilizadas. A colagem com o adesivo tanino comercial apresentaram maior porcentagem de falha na madeira, não diferindo estatisticamente nas três classes de densidade.

\section{DISCUSSÃO}

\subsection{Avaliação das propriedades dos adesivos}

Valores de viscosidade acima de $1500 \mathrm{cP}$ dificultam a aplicabilidade dos adesivos por meios de aspersão [22]. Contudo, o uso desses adesivos é possível quando aplicados manualmente e de forma direta em peças de madeira. Taninos obtidos de Eucalyptus grandis e Eucalyptus pellita apresentaram valores para viscosidade superiores a 6.000 cP [23], assim como extratos tânicos não sulfitados a partir da casca de Eucalyptus grandis $(>10.000 \mathrm{cP})[24]$.

Em comparação com resinas sintéticas, pode-se inferir que extratos de tanino são mais viscosos nas concentrações normalmente requeridas em adesivos [7]. Assim, a alta viscosidade de soluções aquosas de taninos condensados ocorre por ser diretamente proporcional à quantidade de gomas e presença de taninos de alto peso molecular no extrato, suspensões coloidais com acesso lento a todas as partes das moléculas presentes com consequentemente, dificuldade de eliminar ligações de hidrogênio intermoleculares com apenas a diluição.

Para a densidade, resultados diferentes aos encontrados foram observados quando se adicionou tanino comercial de acácia negra à resina sintética [22], proporcionando redução dos valores dessa propriedade, causado pelo tipo de tanino utilizado.

Taninos provenientes da casca de Eucalyptus grandis e Eucalyptus pellita apresentaram valores de $\mathrm{pH}$ na faixa ácida, respectivamente 5,20 e 6,63 [23]. Valores de $\mathrm{pH}$ em faixas ácidas podem acelerar a cura dos adesivos, mudando o comportamento da fase gel [25]. A adição de extrato de tanino Acacia mearnsi em resol fenol-formaldeído induziu a redução do tempo de gel com propriedades finais comparáveis aos obtidos para painéis colados com fenol-formaldeído [26]. No entanto, o tempo de gel pode ser alterado, alterando-se o pH de soluções tânicas [27], promovendo um incremento alcalino, da faixa 4 (24 seg.) para a faixa 10 (>260 seg.), há uma redução de 9,2 vezes no tempo de gel.

A adição crescente de tanino comercial de acácia negra modificado com água (solução 50\%) em adesivos comerciais promoveu um ligeiro aumento no tempo de gel dos adesivos, bem como aumento o teor de sólidos [22], mesmo com um comportamento pouco diferente de outras propriedades das resinas antes da adição do tanino. A matéria-prima utilizada para extração de tanino também pode comprometer a qualidade da solução tânica e consequentemente a eficiência na colagem e alterações na viscosidade do adesivo [22]. A viscosidade de um extrato pode aumentar com o teor de sólidos, especialmente se os hidratos de carbono estiverem presentes na extração [25], pois são responsáveis por boa parte das fortes ligações entre as moléculas.

\subsection{Caracterização química da madeira}

Nos tecidos da madeira, as características químicas variam, de modo que a distribuição é heterogênea, e apresentam quantidades específicas [28] com características bem definidas, que podem ser influenciadas pelo melhoramento genético e ao ambiente que a árvore está submetida.

A quantidade e o tipo de extrativos podem afetar a adesão na superfície da madeira, pois são os principais responsáveis para a inativação da superfície (umedecimento do adesivo), podendo ainda alterar o $\mathrm{pH}$ superficial da madeira. [29]. Em especial neste trabalho, os valores de $\mathrm{pH}$ estão próximos ao relatado pela bibliografia [30], sobretudo para a classe de menor densidade. Valores de extrativos totais baixos, proporcionam colagem mais resistentes, não prejudicando a cura do adesivo [31].

O teor de lignina total encontrado variou de $23,3 \%$ a $30,9 \%$ entre as classes de densidade, de acordo com o encontrado na literatura [16], bem como os valores de holocelulose (67\% a 75\%) [32] para 10 espécies e clones de eucalipto. Para as cinzas, encontrou-se valores pouco inferiores daqueles encontrados por outros autores [33], para madeiras de Eucalyptus globulus e Eucalyptus dunnii, que são mais acumuladoras de minerais do que Eucalyptus saligna e Eucalyptus grandis

De modo geral, a variação da composição química encontrada está próxima de 25 espécies de eucalipto, em foram observadas que a constituição química da madeira era semelhante entre si, com variação de $40 \%$ - 55\% de celulose, $24 \%$ - $40 \%$ de hemicelulose, $18 \%$ - 25\% de ligninas, $1 \%-10 \%$ de extrativos e menos $1 \%$ para teor de cinzas, com densidade básica variando de $0,547 \mathrm{~g} \mathrm{~cm}^{-3}$ a $0,891 \mathrm{~g} \mathrm{~cm}^{-3}$ [34].

$\mathrm{O} \mathrm{pH}$ das diversas espécies situa-se geralmente na faixa entre 3 e 6 , portanto, madeiras com caráter ácido, são mais apropriadas para colagem com adesivos a base de ureia com traços levemente ácido quando 
comparado a adesivos a base de fenol [31]. Para reduzir o pH em nível necessário para a cura ideal do adesivo, madeiras com alta capacidade tampão requerem maiores quantidades de catalisadores ácidos [35].

Maiores quantidades de solução de $\mathrm{NaOH} 5 \mathrm{~g}^{-1}$ foram necessárias elevar o $\mathrm{pH}$ da madeira nas três classes de densidade e modo de extração do que aqueles valores encontrados para Acacia mangium [36], com variação de 0,05 a 0,104 mmol NaOH $5 \mathrm{~g}^{-1}$ dos valores da capacidade tampão ácida, sendo que o pH da madeira de Acacia mangium encontrado estava entre 5,45 e 5,90.

A ureia formaldeído e o fenol formaldeído são as resinas mais utilizadas comercialmente e curam, respectivamente, nos meios ácidos e alcalinos [7]. Por conseguinte, uma madeira de alta acidez possui maior dificuldade de colagem com a resina fenol formaldeído, enquanto madeiras com pH excessivamente ácidos podem causar a pré-cura do adesivo ureia formaldeído durante a fase de fechamento da prensa, prejudicando as propriedades finais do painel.

\subsection{Ensaio de cisalhamento das juntas de madeira coladas}

As propriedades da madeira possuem um nítido efeito nas ligações adesivas e, geralmente, madeiras de folhosas apresentam maiores dificuldades de adesão do que as coníferas [37]. Uma elevada resistência ao cisalhamento das juntas coladas pode ser atribuída à menor porosidade, que dificulta a penetração do adesivo no interior da madeira, contribuindo para a resistência, por limitar a mobilidade do adesivo na estrutura lenhosa. Neste sentido, a ligação adesiva é mais superficial [38].

Nas três classes de densidade, ocorreu heterogeneidade dos valores da resistência ao cisalhamento, que podem ser atribuídos à natureza heterogênea do material, como resíduos de madeira procedentes de diferentes áreas de plantio, diferentes espécies, proporções de cerne e alburno, grã, porosidade e idades [39]. Nas madeiras de média densidade, por abranger maior intervalo de valores de diferente de densidade $(0,50-0,72$ $\mathrm{g} \mathrm{cm}^{-3}$ ) isto foi mais pronunciado.

No material avaliado, por ser procedente de um mix de espécies de eucalipto, vários fatores podem influenciar nos baixos resultados do cisalhamento da linha de cola, tais como: pH, capacidade-tampão e tipos de extrativos da madeira, penetração do adesivo (características anatômicas e densidade da madeira), viscosidade e teor de sólidos do adesivo, tempo e pressão durante a prensagem [37, 40, 5].

$\mathrm{Na}$ classe de densidade alta ocorreu elevado teor de extrativos, e maior resistência ao cisalhamento, similarmente ao encontrado em trabalho em que avaliou-se a qualidade da colagem de madeira de Eucalyptus grandis [29]. Uma correlação positiva entre a resistência ao cisalhamento e o teor de extrativos totais (coeficiente de Pearson de 0,5423), indicou haver influência do teor de extrativos na resistência ao cisalhamento, considerando uma melhor resistência ao cisalhamento aquela madeira com maior teor de extrativos totais.

Linha de cola formada com resorcinol-formaldeído em madeira de Eucalyptus grandis apresentou valores da ordem de 3,81 MPa, 4,72 $\mathrm{MPa}$ e 5,73 $\mathrm{MPa}$ (resistência ao cisalhamento), para madeiras com densidades de $0,55 \mathrm{~g} \mathrm{~cm}^{-3}, 0,55 \mathrm{~g} \mathrm{~cm}^{-3}$ e $0,64 \mathrm{~g} \mathrm{~cm}^{-3}$, na posição radial na região da medula, intermediária e próximo à casca, respectivamente [44]. Confirma-se assim a relação de importância existe entre a densidade e a porosidade da madeira no que se refere a penetração dos adesivos na madeira [40].

Ao considerar que a madeira densa é menos permeável pode-se sugerir composições diferentes de adesivos para madeiras densas, em relação às porosas, objetivando uma penetração ideal de adesivos em cada tipo de madeira [38]. Deste modo, pode se afirmar que a baixa resistência ao cisalhamento nas madeiras com baixa densidade, ocorreu pela excessiva penetração do adesivo na madeira, ocasionando uma ligação mais fraca com linha de cola "faminta".

Para os adesivos naturais, os valores de resistência ao cisalhamento das juntas coladas ficaram abaixo do observado na literatura [41], em foram utilizados tanino obtido a partir da casca de Eucalyptus pellita a 45\% na colagem da madeira de Erisma unsinatum (cedrinho) (resistência média de 5,12 MPa), havendo tendência de acréscimo da resistência tanino desta espécie na proporção de $20 \%$.

A interação adesivo madeira seguiu o mesmo princípio de um trabalho em que foi avaliado a qualidade de adesão em madeira de Corymbia citriodora e Eucalyptus pellita tratadas termicamente onde o adesivo resorcinol-tanino (80:20) proporcionou um desempenho melhor ou semelhante ao adesivo resorcinol puro, sendo mais indicado para as juntas nesta espécie [42].

A resistência ao cisalhamento da madeira de eucalipto em classe de maior densidade, empregando-se ureia formaldeído, tanino industrial, e ureia com tanino (90:10) extraído com carbonato e sulfato de sódio foram similares à literatura [1] em colagem com tanino na madeira de Pinus sp. a temperatura ambiente com valor de 3,69 $\mathrm{MPa}$, com destaque para o adesivo ureia formaldeído com 8,99 MPa de resistência ao cisalhamento. 


\subsection{Falha na madeira das juntas coladas}

As maiores porcentagens de falha no lenho de baixa densidade é indicativo de boa qualidade de adesão, por ocorrer melhor adesão entre a madeira e os adesivos, pois materiais de baixa densidade são mais porosos, proporcionando maior ancoragem do adesivo nas estruturas anatômicas do mesmo [40]. Elevadas falhas na madeira podem estar relacionadas também a sua baixa resistência ao cisalhamento, pois madeira de maior densidade quando submetidas a mesma força, apresentam maior resistência e menores falhas na madeira. Deste modo, não é possível garantir categoricamente a eficiência do processo de adesão em madeiras de menor densidade assim como o afirmado em outros trabalhos em madeira de Eucalyptus sp. [43].

Os valores médios de falha na madeira diferiram entre os adesivos utilizados sendo a madeira colada com ureia formaldeído aquelas com maiores valores de falha (29\% a 62\%), assim como apresentado na literatura [46]. Enquanto que em madeira de Pinus sp. aderida com tanino formaldeído e emulsão polimérica de isocianato, menores valores para falha na linha de cola foram proporcionados pelos adesivos de ureia formaldeído e acetato de polivinila [1], indicando uma baixa eficiência e segurança do mesmo, sendo assim, não sendo indicado para colagem dessa madeira.

\section{CONCLUSÕES}

Os adesivos compostos pelo tanino extraído com sulfato de sódio e com carbonato de sódio puros (100\%) apresentaram características desejáveis para a produção de taninos, mas não o suficiente para sua utilização como adesivo em madeira em sua totalidade, dada a delaminação das lamelas testadas. No entanto, o uso de taninos no enriquecimento de ureia-formaldeído pode ser utilizado na colagem da madeira.

A maior resistência ao cisalhamento na madeira ocorreu na classe de densidade alta, para todos os adesivos empregados, enquanto que as maiores falhas na madeira na maioria dos adesivos testados ocorreram em madeira de baixas densidades.

Os resultados obtidos do processo de colagem, indicam a necessidade de outros estudos com menores intervalos dos valores que abrangem cada classe de densidade, principalmente as de média densidade.

\section{AGRADECIMENTOS}

Os autores agradecem ao empresário Sr. Bruno Bragança pela doação do material utilizado neste estudo; à empresa Seta, pela doação do tanino; à empresa Momentive Química do Brasil Ltda., pela doação do adesivo e à CAPES pela concessão da bolsa de mestrado à primeira autora.

\section{BIBLIOGRAFIA}

[1] GONÇALVES, F.G., SEGUNDINHO, P.G.A., SCHAYDER, L.F., et al., "Avaliação da resistência ao cisalhamento da madeira de Pinus sp. coladas em temperatura ambiente", Ciência da Madeira, v. 7, n. 1, pp. 42-50, Abr. 2016.

[2] MILLER, R.P., "Characteristics and availability of commercially important woods". In: Wood Handbook: Wood as an Engineering Material, Chapter 1, Madison, WI: U.S. Department of Agriculture, Forest Service, Forest Products Laboratory, 1999.

[3] BOA, A.C., GONÇALVES, F.G., OLIVEIRA, J.T.S., et al., "Resíduos madeireiros de eucalipto colados com resina ureia formaldeído à temperatura ambiente”, Scientia Forestalis, v. 42, n. 102, pp. 279-288, Jun. 2014.

[4] MOTTA, J.P., OLIVEIRA, J.T.S., PAES, J.B., et al., “Avaliação da resistência ao cisalhamento de juntas coladas com madeira de teca (Tectona grandis)", Scientia. Forestalis, v. 42, n. 104, pp. 615-621, Dez. 2014.

[5] PIZZI, A., "Natural phenolic adhesives I: Tannin”. In: Pizzi, A., Mittal, K.L. (Org.), Handbook of adhesive technology, 2 ed., chapter 27, New York, Marcel Dekker, pp. 347-358, 2003.

[6] GONÇALVES, C.A., LELIS, R.C.C., "Teores de taninos da casca e da madeira de cinco leguminosas arbóreas", Floresta e Ambiente, v. 8, n. 1, pp. 167- 173, Jan.-Dez. 2001.

[7] PIZZI, A., Advanced wood adhesives technology. New York, Marcel Dekker, 1994.

[8] CARNEIRO, A.C.O., et al., “Adesivos e sua importância na indústria madeireira”, In: Oliveira, J. T. S., et al., (Eds) . Tecnologias aplicadas ao setor madeireiro II, capítulo 4, Vitória, Gráfica Aquarius, 2007.

[9] BRITO, E.O., Produção de chapas de partículas de madeira a partir de maravalhas de Pinus elliottii Engelm. Var. elliottii plantado no sul do Brasil, Tese de D. Sc., UFPR - Universidade Federal do Paraná, Curitiba, PR, Brasil, 1995. 
[10] PAES, J.B., Efeitos da purificação e do enriquecimento do creosoto vegetal em suas propriedades preservativas, Tese. D. Sc., UFV - Universidade Federal de Viçosa, Viçosa, MG, Brasil, 1997.

[11] Associação Brasileira de Normas Técnicas. ABNT. NBR 11941: madeira: determinação da densidade básica. Rio de Janeiro, 2003.

[12] VITAL, B.R., Métodos de determinação da densidade da madeira. Viçosa, SIF, 1984.

[13] MUÑIZ, G.I.B., CORADIN, V.R., Normas de procedimentos em estudo de anatomia da Madeira: IAngiospermae, II- Gimnospermae. Brasília: Laboratório de Produtos Florestais. 15, 1991. Série Técnica.

[14] Technical Association of the Pulp and Paper Industry - TAPPI. T 264 om-88: preparation of wood for chemical analysis. Atlanta, Tappi Technology Park, v.1, 1992b.

[15] GOMIDE, J.L., DEMUNER, B.J. "Determinação do teor de lignina em material lenhoso: método Klarson modificado", O papel, v. 47, n. 8, pp. 36-38, 1986.

[16] GOMIDE, J.L., FANTUZZI NETO, H., REGAZZI, A. J., et al., "Análise de critérios de qualidade da madeira de eucalipto para produção de celulose Kraft”, Revista Árvore, v. 34, n. 2, pp. 339-344, Mar.-Abr. 2010.

[17] Associação Brasileira de Normas Técnicas - ABNT. NBR 7190: projeto de estruturas de madeira. Rio de Janeiro, 1997.

[18] ALMEIDA, V.C., Efeito da adição de carga e extensor nas propriedades do adesivo uréia-formaldeído e dos compensados de Pinus elliottii e Schizolobium amazonicum, Dissertação de M. Sc., UFV - Universidade Federal de Viçosa, Viçosa, MG, 2009.

[19] American Society for Testing and Materials. ASTM D - 2339-98: Standard test method for strength properties of adhesives in two-ply wood construction in shear by tension loading, West Conshohocken, PA, 2011.

[20] ACOSTA, F.M., PÉREZ, P.M. "Evaluación de una unión laminada con adhesivo tipo PVA y maderas de plantación: Acacia mangium, Tectona grandis y Terminalia amazonia", Revista Forestal Venezoelana, v. 55, n. 2, pp.145-153, 2011.

[21] American Society for Testing and Materials. ASTM D - 5751-99: Standard specification for adhesives used for laminate joints in nonstructural lumber products, West Conshohocken, PA, 2012.

[22] GONÇALVES, F.G., LELIS, R.C.C., "Propriedades de duas resinas sintéticas após adição de tanino modificado", Floresta e Ambiente, v. 16, n. 2, pp. 01 - 07, 2009.

[23] VITAL, B.R., CARNEIRO, A. C. O., PIMENTA, A. S., et al., "Adesivos à base de taninos das cascas de duas espécies de eucalipto para produção de chapas de flocos”, Revista Árvore, v. 28, n. 4, pp. 571-582, Jul.-Ago. 2004.

[24] CARNEIRO, A.C.O., VITAL, B.R., FREDERICO, P.G.U., et al., "Efeito da hidrólise ácida dos taninos de Eucalyptus grandis W. Hill ex Maiden nas propriedades dos adesivos tânicos", Revista Árvore, v. 33, n. 4, pp. 733-739, Jul.-Ago. 2009.

[25] DUNKY, M., “Adhesives in the Wood Industry”, In: Pizzi, A., Mittal, K.L. (Org.). Handbook of adhesive technology, 2 ed., chapter 47, New York, Mareei Dekker, 2003.

[26] STEFANI, P.M., PENA, C., RUSECKAITEA, R.A., et al., "Processing conditions analysis of Eucalyptus globulus plywood bonded with resol-tannin adhesives", Bioresource Technology, v. 99, n. 13, pp. 59775980, Sep. 2008.

[27] HOONG, Y.B., PARIDAH, M.T., LUQMAN, C.A., et al., "Fortification of sulfited tannin from the bark of Acacia mangium with phenol-formaldehyde for use as plywood adhesive", Industrial Crops and Products, v. 30, n. 3, pp. 416-421, Nov. 2009.

[28] TREVISAN, H., TIEPPO, F.M.M., CARVALHO, A.G., et al., “Avaliação de propriedades físicas e mecânicas da madeira de cinco espécies florestais em função da deterioração em dois ambientes”, Revista Árvore, v. 31, n. 1, pp. 93-101, Jan.-Fev. 2007.

[29] ALBINO, V.C.S., MORI, F. A., MENDES, L. M., et al., "Influência das características anatômicas e do teor de extrativos totais da madeira de Eucalyptus grandis W. Hill ex Maiden na qualidade da colagem", $C i$ ência Florestal, v. 22, n. 4, pp. 803-811, Out.-Dez.2012.

[30] SILVÉRIO, F.O., BARBOSA, L.C.A., GOMIDE, J.L., et al., "Metodologia de extração e determinação do teor de extrativos em madeiras de eucalipto", Revista Árvore, v. 30, n. 6, pp. 1009-1016, Nov.-Dez. 2006. [31] ALBUQUERQUE, C.E.C., IWAKIRI, S., KEINERT JUNIOR, S. “Adesivos e adesão”, In: Iwakiri, S. (ed.). Painéis de madeira reconstituída, capítulo 1, Curitiba: FUPEF, 2005. 
[32] ANDRADE, M.C.N., MINHONI, M.T.A., SANSÍGOLO, C.A., et al., "Estudo comparativo da constituição nutricional da madeira e casca de espécies e clones de eucalipto visando o cultivo de Shiitake em toras", Revista Árvore, v. 35, n. 2, pp. 183-192, Mar.-Abr. 2011.

[33] FOELKEL, C., Casca da árvore do eucalipto: aspectos morfológicos, fisiológicos, florestais, ecológicos e industriais, visando a produção de celulose e papel. 2005. 109p. http://www.eucalyptus.com.br. Acessado em maio de 2016.

[34] MORI, F.A., LOPES, C., MORI, S.O., et al., "Influência do sulfito e hidróxido de sódio na quantificação em taninos da casca de barbatimão (Stryphnodendron adstringens)", Floresta e Ambiente, v. 10, n. 1, pp. 86-92, Jan.-Jul. 2003.

[35] MALONEY, T.M., Modern particleboard \& dry-process fiberboard manufacturing. San Francisco, Miller Freeman, 1993.

[36] GONÇALVES, F.G., LELIS, R.C.C., "Caracterização tecnológica da madeira de Acacia magium Willd em plantio consorciado com eucalipto”, Floresta e Ambiente, v. 19, n. 3, pp. 286-295, Jul.-Set. 2012.

[37] KOLLMANN, F.F.P., et al., Principles of wood science and technology. Berlin, Springer-Verlag, 1975. v. 2.

[38] MARRA, A.A., Technology of wood bonding. Nova York, Van Nostrand Reinhold, 1992.

[39] ALBUQUERQUE, C.E.C., LATORRACA, J.V.F., "Influência das características anatômicas da madeira na penetração e adesão de adesivos”, Floresta e Ambiente, v. 7, n. 1, pp. 158-166, Jan.-Fev. 2000.

[40] IWAKIRI, S., Painéis de madeira. Curitiba, FUPEF, 1998.

[41] TEODORO, A.S., LELIS, R.C.C., "Colagem de madeira sólida com adesivo natural à base de tanino", Revista de Ciências da Vida, v. 25, n. 1, pp. 55-59, Jan.-Jun. 2005.

[42] NUNES, C.S., NASCIMENTO, A.M., GARCIA, R. A., et al., "Qualidade de adesão das madeiras de Corymbia citriodora e Eucalyptus pellita tratadas termicamente”, Scientia Forestalis, v. 44, n. 109, pp. 4156, Mar. 2016.

[43] PLASTER, O.B., OLIVEIRA, J.T.S., ABRAHAO, C.P., et al., "Comportamento de juntas coladas da madeira serrada de Eucalyptus sp", Cerne, v. 14, n. 3, pp. 251-258, Jul.-Set. 2008.

[44] ALBINO, V.C.S., MORI, F.A., MENDES, L.M., et al., "A study of the bonding of wood from Eucalyptus grandis W. Hill ex Maiden using resorcinol-formaldehyde adhesive", Cerne, v. 16, n. 4, pp. 443-449,

Oct.-Dec. 2010.

[45] GOLDSCHIMILD, O., "Ultraviolet spectra", In: Sarkanen, K.V., Luswig, C.H. (Eds.). Lignins, New York, Wiley Interscience, pp. 241-246, 1971.

[46] LIMA, C.K.P., MORI, F.A., MENDES, L.M., et al., "Colagem da madeira de clones de Eucalyptus com três adesivos comerciais”, Scientia Forestalis, v. 36, n. 77, pp. 73-77, Mar. 2008.

[47] PAES, J.B., DINIZ, C.E.F., LIMA, C.R., et al., "Taninos condensados da casca de angico-vermelho (Anadenanthera colubrina var. cebil) extraídos com soluções de hidróxido e sulfito de sódio", Revista Caatinga, v. 26, n. 3, pp. 22-27, Jul.-Set. 2013.

[48] AZEVÊDO, T.K.B., PAES, J.B., CALEGARI, L., et al., Qualidade dos taninos de jurema-preta (Mimosa tenuiflora) para a produção de adesivo tanino formaldeído, Ciência Florestal, Santa Maria, v. 25, n. 2, pp. 507-514, Abr.-Jun. 2015. 